\title{
Effects of prophylactic use of brimonidine $0.2 \%$ on intraocular pressure after YAG-capsulotomy
}

\author{
Tayyaba Gul Malik ${ }^{1}$, Aalia Ali $^{2}$, Syed Abdullah Mazhar ${ }^{2}$, Rabail Alam \\ ${ }^{1}$ Ameer ud din Medical College/Lahore General Hospital, Lahore, Pakistan \\ ${ }^{2}$ Arif Memorial Hospital, Lahore, Pakistan \\ ${ }^{3}$ Institute of molecular Biology and Biotechnology, University of Lahore, Lahore, Pakistan
}

\begin{abstract}
BACKGROUND: Rise in intraocular pressure (IOP) is the commonest complication after YAG posterior capsulotomy. As there are different opinions regarding use of anti-glaucoma therapy before YAG, we compared post-YAG IOP between the patients who had Brimonidine eye drops and those who did not have any anti-glaucoma treatment. MATERIAL AND METHODS: It was a prospective study that included patients who had undergone uneventful phacoemulsification with foldable intraocular lens implantation and YAG posterior capsulotomy. One hundred fifty patients were divided into two groups; (a group with prophylactic brimonidine $0.2 \%$ eye drops before laser and a group without any anti-glaucoma therapy). Intraocular pressure was checked pre-laser and one hour after laser procedure. RESULTS: Out of 150 patients, 78 were in brimonidine group and 72 in the control group. The mean age of the patients was $60.39 \pm 12.98$ years. In the brimonidine group, IOP was $12.56 \pm 2.38 \mathrm{~mm} \mathrm{Hg}$ and $12.29 \pm 3.64 \mathrm{~mm}$ $\mathrm{Hg}$ before and after YAG, respectively. In the control group, IOP was $12.24 \pm 1.53 \mathrm{~mm} \mathrm{Hg}$ and $13.38 \pm 2.84 \mathrm{~mm}$ $\mathrm{Hg}$ before and after YAG. Brimonidine $0.2 \%$ caused a decrease in IOP, but the post-laser IOP difference between the two groups was not statistically significant. The change in IOP before and after using brimonidine $0.2 \%$ was also not statistically significant.

CONCLUSION: Every patient undergoing YAG capsulotomy does not require prophylactic anti-glaucoma therapy. Only the patients prone to high IOP, glaucoma suspects, and diagnosed cases of glaucoma should be given prophylactic treatment.
\end{abstract}

KEY WORDS: glaucoma; intraocular pressure; YAG capsulotomy; brimonidine; trabeculitis

Ophthalmol J 2021; Vol. 6, 265-269

\section{INTRODUCTION}

The rise in intraocular pressure (IOP) is the commonest complication after YAG posterior capsulotomy. Various mechanisms for this rise in intraocular pressure (IOP) are described [1]. The commonest theory is the trabecular meshwork blockage caused by debris deposition. Trabeculitis secondary to the YAG laser shock waves and post-laser inflammation are other major contributing factors. Inflammatory edema of the ciliary body or iris root can also result in angle-closure glaucoma in some cases [1].

The extent to which the IOP rises depends on several factors; the greater the energy used for capsulotomy, the greater is the pressure rise. The thickness of the capsule is also proportional to the increase in IOP. Similarly, the size of capsulotomy 
(the larger the size of capsulotomy, the greater is the amount of debris) and an in-experienced surgeon using high energy can also contribute to IOP rise. Many surgeons have adopted the use of prophylactic anti-glaucoma drugs to avoid this rise of IOP. However, other reports indicated either no significant increase in IOP or IOP spike despite prophylactic use of pressure-lowering drugs [2].

Different medications have been tried to prevent the increase in IOP after YAG capsulotomy. Brimonidine 0.2\%, Apraclonidine, Acetazolamide, and Bimatoprost eye drops have been used for this purpose [3]. In contrast to these reports, some authors did not report any rise of IOP after YAG posterior capsulotomy, in non-glaucomatous pseudophakic eyes [4].

As there were different opinions regarding the use of prophylactic anti-glaucoma therapy in patients requiring YAG capsulotomy, we decided to find out the rise of IOP in eyes, which underwent YAG capsulotomy in our setup. We also compared the increase in IOP between those who had prophylactic anti-glaucoma medicine and those who did not have prophylactic treatment. The study's rationale was to avoid using anti-glaucoma drugs in patients who do not have the tendency of a significant rise in IOP after YAG capsulotomy. This will save the patients from unnecessary use of a prophylactic anti-glaucoma drug in healthy non-glaucomatous eyes.

\section{MATERIAL AND METHODS}

It was a quasi-experimental study performed at Arif Memorial teaching hospital, Lahore, and Lahore General Hospital (Pakistan) from January 2020 to October 2021. We followed the tenets of the Declaration of Helsinki for this study and obtained informed consent from the patients before the procedure. The ethical review board approved the study protocol.

Sample size was calculated using calculator.net [confidence interval $(\mathrm{CI})=95 \%$, margin of error $=5 \%$, population proportion $=5.7 \%]$. The sample size came out to be 83 . Patients who had undergone uneventful phacoemulsification with foldable intraocular lens implantation and visiting the hospital for YAG posterior capsulotomy were included. One hundred fifty patients met the inclusion criteria from January 2020 to October 2021. The ideal situation of only one surgeon performing the procedure could not be adopted because of two-center research. Patients who had a history of or the clinical examination suggestive of complications during cataract surgery or in the postoperative period were excluded. Patients with glaucoma, glaucoma suspects, patients with IOP $\geq 20 \mathrm{~mm} \mathrm{Hg}$, corneal opacities, retinal pathologies, uveitis, optic neuropathy, and history of any other intraocular surgery were also excluded.

A complete history was taken, and an ocular examination was performed on all patients. Pre-laser IOP was checked using Goldmann Applanation Tonometer. The patients were divided into two groups. One group received prophylactic brimonidine $0.2 \%$ eye drops one hour before laser treatment. The other group did not receive any anti-glaucoma therapy. Intraocular pressure was checked pre-laser procedure and one hour after it. There were 72 patients in the control group and 78 patients in the brimonidine group. Pupils were dilated using tropicamide eye drops and phenylephrine eye drops. Capsulotomy size was kept at $4 \mathrm{~mm}$ and energy was not more than $80 \mathrm{~mJ}$. After the procedure, prednisolone $1 \%$ eye drops were prescribed three times a day for seven days. Repeated measure ANOVA was used for data analysis.

\section{RESULTS}

A total of 150 patients fulfilled the inclusion criteria. There were 78 patients in the brimonidine group and 72 in the control group. Table 1 shows the demographic details of the two groups.

\section{RESULTS}

The mean age of the patients was $60.39 \pm 12.98$ years. The mean age of males was $61.31 \pm 11.52$, and females was $59.72 \pm 13.95$ years. Visual acuity of both groups ranged from finger counting to $6 / 12$. In the brimonidine group, IOP was $12.56 \pm 2.38 \mathrm{~mm} \mathrm{Hg}$ before YAG, and post-YAG was $12.29 \pm 3.64 \mathrm{~mm} \mathrm{Hg}$ with a mean fall of $0.27 \pm 1.26$ after laser treatment. In the control group, IOP was $12.24 \pm 1.53 \mathrm{~mm} \mathrm{Hg}$ before YAG, and post-YAG was $13.38 \pm 2.84 \mathrm{~mm}$ $\mathrm{Hg}$ with a mean rise of $1.14 \pm 1.31 \mathrm{~mm} \mathrm{Hg}$. Although the results showed that brimonidine $0.2 \%$ caused a decrease in IOP, the post-laser IOP difference was not statistically significant. The results showed no statistically significant difference between the IOP of the two groups before and after laser procedure (Tab. 2, 3). 
Table 1. Demographic characteristics of the studied groups

\begin{tabular}{|l|c|c|c|}
\hline \multicolumn{2}{|c|}{ Brimonidine group } & Control group & Total eyes \\
\hline Gender & 30 & 32 & 62 \\
\hline Male & 48 & 40 & 88 \\
\hline Female & 78 & 72 & 150 \\
\hline Total & \multicolumn{3}{|c|}{} \\
\hline Treated Eye & 42 & 40 & 82 \\
\hline Left & 36 & 32 & 68 \\
\hline Right & 78 & 72 & 150 \\
\hline Total & & & \\
\hline
\end{tabular}

Table 2. No statistically significant difference in intraocular pressure (IOP) at one hour after laser procedure in the brimonidine group as compared to the control group

\begin{tabular}{|c|c|c|c|c|c|}
\hline \multicolumn{6}{|c|}{ Tests of between-subjects effects } \\
\hline \multicolumn{6}{|l|}{ Measure } \\
\hline \multicolumn{6}{|c|}{ Transformed Variable } \\
\hline Source & $\begin{array}{l}\text { Type III sum of } \\
\text { squares }\end{array}$ & df & Mean square & $\mathbf{F}$ & Sig. \\
\hline Intercept & 47684.137 & 1 & 47684.137 & 4852.747 & 0.000 \\
\hline group & 10.590 & 1 & 10.590 & 1.078 & 0.301 \\
\hline Error & 1454.280 & 148 & 9.826 & & \\
\hline
\end{tabular}

Table 3. Statistically insignificant decrease in intraocular pressure (IOP) in the brimonidine group. There was no significant variance before and after laser procedure in brimonidine group

\begin{tabular}{|l|l|c|c|c|c|c|}
\hline \multicolumn{2}{|l|}{ Tests of Within-Subjects Contrasts } \\
\hline Measure \\
\hline Source & $\begin{array}{c}\text { Type III sum } \\
\text { of squares }\end{array}$ & df & Mean square & F & Sig. \\
\hline Time & Linear & 14.158 & 1 & 14.158 & 2.828 & 0.095 \\
\hline Time * group & Linear & 37.118 & 1 & 37.118 & 7.414 & 0.007 \\
\hline Error (time) & Linear & 740.979 & 148 & 5.007 & & \\
\hline
\end{tabular}

\section{DISCUSSION}

This particular research showed no statistically significant difference of post-laser IOP between the bromonidine group and the control group at one hour after laser therapy. Similarly, we did not find any statistically significant rise of IOP in both groups after laser, although the brimonidine group showed a decrease in IOP after YAG capsulotomy. Our results were consistent with the results of Shani et al., who did not find any significant rise in IOP after YAG laser capsulotomy [4]. Similarly, Ari et al., in their study of healthy pseudophakic eyes, did not find any persistent rise of IOP after YAG posterior capsulotomy [5].
Contrary to this, Thiab recently published a study where he mentioned a statistically significant $(\mathrm{p}<0.001)$ rise in IOP at one hour after laser treatment. There was an average rise of $8.35 \mathrm{~mm}$ $\mathrm{Hg}$ from baseline measurement. However, the author did not mention the average amount of energy used for the laser procedure. Such a significant rise of IOP in Thiab's study could be due to the high energy [6]. In our control group, the mean increase of IOP was $1.14 \pm 1.31 \mathrm{~mm} \mathrm{Hg}$. In this particular study, we kept the maximum energy below $80 \mathrm{~mJ}$.

To see the effects of different energy levels on IOP, Parajuli et al. divided 96 eyes of 83 patients into two groups. The group with a mean energy of 
$26.64 \pm 12.92 \mathrm{~mJ}$ and another group with energy of $81.96 \pm 32.10 \mathrm{~mJ}$. Intraocular pressure was raised in both groups irrespective of the energy used. However, the IOP did not come to the pre-laser levels one month after treatment in the high-energy group. It is important to note that the rise in IOP did not require any anti-glaucoma therapy in his study [7].

As multiple factors are involved in the rise of IOP after YAG capsulotomy, some researchers have studied the effect of the size of capsulotomy on IOP. It was observed that IOP was higher in patients with large capsulotomies than the small ones. This can be explained by the amount of debris produced in making large capsulotomy more than the small-sized capsulotomy holes [8]. The thickness of the posterior capsule also affects the IOP as thick capsules require a more significant amount of energy for the capsulotomy.

Considering the rise in IOP after YAG capsulotomy, which can be devastating in glaucomatous eye, different anti-glaucoma therapies have been tried. Apraclonidine 1\% eye drops were common practice in specific setups to avoid an inadvertent rise in IOP. Achiron performed a survey revealing that $71 \%$ of the ophthalmologists prescribed anti-glaucoma therapy to the YAG capsulotomy patients [9]. However, he did not consider the association of a number of laser shots, laser energy, diabetes, hypertension, and glaucoma status with post-laser IOP. He believed that using prophylactic apraclonodine $1 \%$ eye drops was unnecessary to measure IOP in every patient except for the high-risk patients. In contrast to this, we found in our study that the rise of IOP was not statistically significant after YAG capsulotomy. However, clinically speaking, a small amount of increase becomes significant in glaucoma suspects or who have chances of optic nerve damage.

Although in the majority of cases, an increase in IOP is not very significant with or without the use of anti-glaucoma therapy. However, in one case, an IOP elevation of $60 \mathrm{~mm} \mathrm{Hg}$ was recorded by 24 hours, even after using apraclonidine $1 \%$ prophylaxis [10].

Some authors have compared the effects of apraclonidine $1 \%$ eye drops with brimonidine $0.2 \%$ eye drops to lower IOP in patients undergoing YAG capsulotomy. Chen found that a single drop of brimonidine $0.2 \%$ given pre-laser was comparable to the effects of apraclonidine $0.5 \%$ to prevent IOP elevation immediately after laser [11].

Rao et al. noted significant spikes of high IOP in patients who underwent YAG capsulotomy. They recommended the use of timolol $0.5 \%$ one hour before laser procedure in all patients and oral acetazolamide with topical timolol in patients with high IOP even after timolol prophylaxis [12]. Other authors have also shown comparable results with the use of brimonidine $0.2 \%$ [13].

Different regimens have been described in the literature to prevent high IOP after YAG. The commonest one is the use of a drop of $0.2 \%$ brimonidine, 1-hour pre-laser treatment, and one drop immediately after it [14].

Ümit Çalli et al. used brimonidine $0.2 \%$ eye drops one hour before laser treatment (similar to our regimen). They also stressed the importance of the number of shots and the total energy used for the capsulotomy [15]. In our study, the total energy was less than $80 \mathrm{~mJ}$ but no record of the shots was kept. In our view, the total amount of energy is more important than the number of shots.

Another protocol used by Saleem et al. was to instill $0.2 \%$ brimonidine immediately after laser treatment and then twice a day $(B D)$ for seven days [16]. Authors used this regimen to prevent the persistent increase of IOP, which is rarely encountered. According to another research, the mean rise of IOP was $8.76 \mathrm{~mm} \mathrm{Hg}$ after YAG capsulotomy [17]. Patients were treated with brimonidine. On the third post-laser day, one patient still had high IOP despite brimonidine use.

Yellamelli et al. compared brimonidine 0.2\% with timolol eye drops. Brimonidine had shown better results than timolol in lowering IOP $(1.83 \mathrm{~mm}$ $\mathrm{Hg}$ compared to $1.53 \mathrm{~mm} \mathrm{Hg}, \mathrm{p}=0.05$ ) [18].

Variability in the results shown by different authors could be due to the difference in measuring IOP. Manandhar et al. noted that mean IOP was $12.73 \pm 3.3 \mathrm{~mm} \mathrm{Hg}$ at one hour and $11.98 \pm 3.2 \mathrm{~mm} \mathrm{Hg}$ at two hours after YAG [19].

Using anti-glaucoma prophylaxis becomes important if the capsule is thick and high total energy is used for the capsulotomy. Murtaza et al. showed that $62 \%$ of the treated patients had increased IOP when high energy was used [20]. Thus, it supports the idea that anti-glaucoma prophylaxis may not be necessary for all patients undergoing YAG capsulotomy. The number of shots, power, total energy - all adversely affect the IOP. The same protocol cannot be applied to all patients - one shoe does not fit all.

Limitations of this study were that we did not compare the effects of energy on the IOP, but we kept the maximum energy less than $80 \mathrm{~mJ}$. Our fol- 
low-up was of short duration, and longer follow-ups with and without the use of anti-glaucoma therapy should be done to analyze the later rise of IOP.

\section{CONCLUSION}

Our results did not show any additional benefit of using anti-glaucoma prophylaxis in every patient undergoing YAG capsulotomy. As we had excluded glaucoma patients and glaucoma suspects, we found that the rise in IOP was mild in our study (mean rise of $1.14 \pm 1.31 \mathrm{~mm} \mathrm{Hg}$ ). A mild elevation of IOP $<5 \mathrm{~mm} \mathrm{Hg}$ in otherwise normal eyes can be overlooked. However, in high-risk patients with pre-operative borderline IOP, a large cup/disc ratio and in diagnosed glaucoma patients the risk of IOP elevation is higher. These patients can benefit from using anti-glaucoma therapy. It should also be noted that in high-risk cases, single drug use will not be of any benefit if the pressures continue to be raised in the following days. Thus, the role of IOP monitoring in these cases is more important than giving prophylactic anti-glaucoma therapy before YAG laser capsulotomy. A single spike of IOP can lead to devastating results in advanced glaucoma.

Our study showed no need to use anti-glaucoma therapy in every patient undergoing laser capsulotomy. Patients prone to high IOP, glaucoma suspects, and diagnosed cases of glaucoma should be given prophylactic treatment, and they also need long-term follow-up.

\section{REFERENCES}

1. MacEwen CJ, Dutton GN. Neodymium-YAG laser in the management of posterior capsular opacification--complications and current trends. Trans Ophthalmol Soc U K. 1986; 105 ( Pt 3): 337-344, indexed in Pubmed: 3466457.

2. Lin JC, Katz LJ, Spaeth GL, et al. Intraocular pressure control after $\mathrm{Nd}$ :YAG laser posterior capsulotomy in eyes with glaucoma. $\mathrm{Br} \mathrm{J}$ Ophthalmol. 2008; 92(3): 337-339, doi: 10.1136/bjo.2007.125310, indexed in Pubmed: 18211944.

3. Minello AA, Prata Junior JA, Mello PA. [Efficacy of topic ocular hipotensive agents after posterior capsulotomy]. Arq Bras Oftalmol. 2008; 71(5): 706-710, doi: 10.1590/s0004-27492008000500018, indexed in Pubmed: 19039468.

4. Shani L, David R, Tessler Z, et al. Intraocular pressure after neodymium:YAG laser treatments in the anterior segment. $J$ Cataract Refract Surg. 1994; 20(4): 455-458, doi: 10.1016/s08863350(13)80184-8, indexed in Pubmed: 7932138.
5. Ari S, Cingü AK, Sahin A, et al. The effects of Nd:YAG laser posterior capsulotomy on macular thickness, intraocular pressure, and visual acuity. Ophthalmic Surg Lasers Imaging. 2012; 43(5): 395-400, doi: 10.3928/15428877-20120705-03, indexed in Pubmed: 22785102.

6. Thiab HH. Changes in intraocular pressure after ND-yag laser posterior capsulotomy. Int J Clin Exp Ophthalmol. 2020; 4(1): 021-030, doi: 10.29328/journal.ijceo.1001029.

7. Parajuli A, Joshi P, Subedi $P$, et al. Effect of Nd:YAG laser posterior capsulotomy on intraocular pressure, refraction, anterior chamber depth, and macular thickness. Clin Ophthalmol. 2019; 13: 945-952, doi: 10.2147/OPTH.S203677, indexed in Pubmed: 31239636.

8. Karahan E, Tuncer I, Zengin MO. The Effect of ND:YAG Laser Posterior Capsulotomy Size on Refraction, Intraocular Pressure, and Macular Thickness. J Ophthalmol. 2014; 2014: 846385, doi: 10.1155/2014/846385, indexed in Pubmed: 24724016.

9. Achiron A. Intraocular Pressure Spikes following Neodymium-doped Yttrium Aluminum Garnet Laser Capsulotomy: Current Prevalence and Management in Israel. J Curr Glaucoma Pract. 2017; 11(2): 63-66, doi: 10.5005/jp-journals-10028-1225, indexed in Pubmed: 28924341.

10. Nesher $R$, Kolker AE. Failure of apraclonidine to prevent delayed IOP elevation after Nd:YAG laser posterior capsulotomy. Trans Am Ophthalmol Soc. 1990; 88: 229-232 (discussion 233-236).

11. Chen TC, Ang RT, Grosskreutz CL, et al. Brimonidine $0.2 \%$ versus apraclonidine $0.5 \%$ for prevention of intraocular pressure elevations after anterior segment laser surgery. Ophthalmology. 2001; 108(6): 1033-1038, doi: 10.1016/s0161-6420(01)00545-0, indexed in Pubmed: 11382625.

12. Rao CMS, Satyasrinivas V, Muralikrishna V, et al. Clinical Study of Visual Outcome and Intraocular Pressure Changes Following Neodymium-doped Yttrium Aluminum Garnet Laser Capsulotomy in Post-operative Cataract Patients with Posterior Capsule Opacification. . Intern J Sci Study. 2017; 5: 76-82, doi: 10.17354/ijss/2017/558.

13. Singhal $D$, Desai $R$, Desai $S$, et al. Use of topical brimonidine to prevent intraocular pressure elevations following Nd: YAG-laser posterior capsulotomy. J Pharmacol Pharmacother. 2011; 2(2): 104-106, doi: 10.4103/0976-500X.81902, indexed in Pubmed: 21772770.

14. Seong GJ, Lee YG, Lee JH, et al. Effect of $0.2 \%$ brimonidine in preventing intraocular pressure elevation after Nd:YAG laser posterior capsulotomy. Ophthalmic Surg Lasers. 2000; 31(4): 308-314, indexed in Pubmed: 10928668.

15. Calli U. The effect of prophylactic topical brimonidine on intraocular pressure after Nd: YAG laser posterior capsulotomy. J Kartal Train Res Hosp. 2016, doi: 10.5505/jkartaltr.2015.066933.

16. Saleem M, Manzoor M, Shehzad A, et al. Brimonidine efficacy on intraocular pressure control following ND: YAG laser capsulotomy. Pak Armed Forces Med J. 2018; 68(2): 245-249.

17. Niazi $M$, Rauf $A$, Nadeem Y. Brimonidine for Prevention of Intraocular Pressure Elevation after YAG-Posterior Capsulotomy. Pak J Ophthalmol. 2020; 36(2), doi: 10.36351/pjo.v36i2.1006.

18. Yellamelli A, Baldev V, Batra N, et al. Effect of topical brimonidine $0.15 \%$ versus timolol $0.5 \%$ to prevent intraocular pressure elevation after nd: yag laser capsulotomy. J Evid Based Med Healthc. 2017; 4(55): 3365-3368, doi: 10.18410/jebmh/2017/668.

19. Manandhar LD, Gurung N, Shrestha K, et al. Study of Intraocular Pressure following Neodynium Yttrium Aluminium Garnet Laser Capsulotomy with the Use of Brimonidine: A Descriptive Crosssectional Study. JNMA J Nepal Med Assoc. 2021; 59(233): 15-18, doi: 10.31729/jnma.5130, indexed in Pubmed: 34508462.

20. Murtaza B, Hussain AW, Haq AU, et al. Changes in intraocular pressure following high energy Nd-Yag laser posterior capsulotomy. Pak Armed Forces Med J. 2018; 68: 872-875. 